\title{
SYMBOL-ASYNCHRONOUS TRANSMISSION IN MULTIBEAM SATELLITE USER DOWN-LINK: RATE REGIONS FOR NOVEL SUPERPOSITION CODING SCHEMES
}

\author{
Nele Noels ${ }^{\star}$ Marc Moeneclaey ${ }^{\star}$ Tomás Ramírez $^{\dagger}$ Carlos Mosquera $^{\dagger}{\text { Màrius } \text { Caus }^{\$} \text { Adriano Pastore }}^{\$}$ \\ * Digital communications and information processing group, Ghent University, Ghent, Belgium \\ $\dagger$ atlanTTic research center, University of Vigo, Galicia, Spain \\ $\$$ Centre Tecnològic de Telecommunicacions de Catalunya (CTTC/CERCA), \\ Castelldefels, Barcelona, Spain
}

\begin{abstract}
We consider the forward link of a multibeam satellite system with high spectral reuse and the novel low-complexity transmission and detection strategies from [1]. More specifically, we study the impact of a time offset between the antenna beams that cooperate to simultaneously serve a given user. Assuming Gaussian signaling, we provide closed-form expressions for the achievable rate region. It is demonstrated that, in the absence of timing information at the gateway, this region is not affected by a time offset. Our numerical results further show that, in case timing is known at the gateway, an offset of half a symbol period at both user terminals is optimal in terms of spectral efficiency.
\end{abstract}

Index Terms-Satellite communication downlink, Multiplexing, Asynchronous transfer mode, Radiofrequency interference, Mutual Information

\section{INTRODUCTION}

In search of novel technologies to meet the very high throughput demands of the envisaged integrated satellite-terrestrial communication networks of the future (such as $5 \mathrm{G}$ ), the satellite community is looking at multibeam satellite communication systems with a frequency plan that is characterized by a very high level of spectral reuse [2]. In such systems co-channel interference, caused by neighboring beams that are transmitting within the same frequency band, is a major issue and interference management strategies are indispensable. The forward link of a multibeam satellite system where adjacent spot beam areas share the same frequency plan and polarization is shown in Fig. 1.

With respect to the forward link, the interference management strategy that has received most of the attention so far is precoding at the gateway $(\mathrm{GW})$. The precoding strategy basically consists in reducing the interference at the user terminals (UT) by predistorting the signal at the GW. The UTs then simply treat the residual interference as noise such that conventional demodulation techniques can be applied. Hence, all additional complexity is situated at the GW. Unfortunately, a massive increase in the amount of return signaling results from the fact that each of the UTs needs to quasicontinuously report to the $\mathrm{GW}$ about changes in the estimates (1 per beam) of the channel coefficients and the time offsets (TO). The use of inaccurate or outdated estimates can cause a significant performance degradation.

The last couple of years, multiuser detection (MUD) at the UTs has gained interest as a potential alternative for GW precoding [3]-[6]. The MUD strategy consists in jointly decoding the interference and the useful signal at the UTs. Since, by definition, the interference component does not contain useful information it is subsequently disregarded. As opposed to precoding at the GW, the technique leaves the GW transmission scheme unaltered. Hence, channel estimation and synchronization is, strictly speaking, only

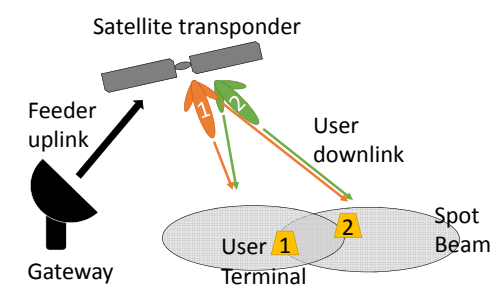

Fig. 1. Forward link of a multibeam satellite system where adjacent spot beam areas share the same frequency plan and polarization.

required at the UTs and there is no need for additional return signaling. ${ }^{1}$ However, a major disadvantage of MUD is that it causes a large increase in UT complexity.

Only recently, a third approach has been proposed in [1]. The idea is to depart from the two extremes of precoding at the GW and MUD at the UTs and to develop novel low-complexity transceiver schemes that allow to exploit the co-channels interference to the users maximum advantage. The proposed strategies are based on splitting the user data streams in multiple substreams that are first independently encoded (each with their own rate) and then linearly combined (with different weights) to generate the different beam waveforms. The weight factors are selected to maximize a convenient metric (e.g., the system sum-rate) under given channel amplitude conditions and fixed per-beam power constraints. The UTs apply a convenient form of successive cancellation decoding (SCD) [7]. ${ }^{2}$ It is clear that, at both sides of the communication link, the processing is modified. However, because the different substreams are independently encoded, the increase in complexity remains moderate. Also, the return signaling requirements are significantly relaxed as compared to precoding. This is because it suffices to feed back variations in the estimates of the real-valued channel amplitudes only and because the amplitude of a fixed satellite channel typically varies very slowly with time, especially as compared to the speed of the channel phase variations [9]. Assuming synchronous waveform reception and convenient user pairing, the potential of these schemes has been demonstrated [1]. However, with expected interbeam differential delays in the order of a few nanoseconds and tentative baud rates up to 1 Tbps [10],

\footnotetext{
${ }^{1}$ Although in many publications on MUD synchronous waveform reception is assumed.

${ }^{2}$ The approach is somewhat related to the work in [8], although considerably different given the fact that partial precoding at the $\mathrm{GW}$ is not being considered.
} 


\section{mi!}

Fig. 2. 2-Color spectral re-use scheme for the spot beam areas of a multibeam satellite system.

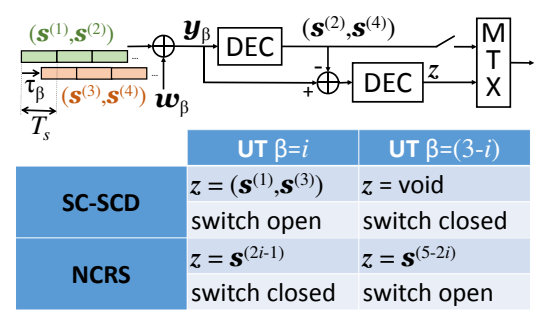

Fig. 3. Summary of SCD strategies at UTs $\beta=1,2$ in SC-SCD and NCRS when $\left(\boldsymbol{s}^{(2)}, \boldsymbol{s}^{(4)}\right)$ is allocated to user $i=1,2$.

the reality of asynchronous waveform reception cannot just be a priori ignored. In some cases, the TO between the interfering waveforms that arrive at a given UT can be several symbol intervals long [9], [11]. In this paper, we investigate the implications of such TOs on the achievable rate regions of the schemes in [1]: superposition coding with successive interference decoding (SC$\mathrm{SCD}$ ) and non coherent rate splitting (NCRS). It is shown that, if no TO information is fed back to the GW, the achievable rate region is the same as for zero TOs. On the other hand, if the GW knows the TOs, the most beneficial TO is $50 \%$ of a symbol period.

The outline of the paper is as follows. Section II introduces the system model and the SC-SCD and NCRS transceiver strategies. Section III is devoted to computing the rate region of SC-SCD and NCRS in case there is a TO between the cooperating beams. Section IV presents numerical results. Section V summarizes the main conclusions and indicates some potential ideas for future work.

\section{SYSTEM MODEL AND TRANSCEIVER STRATEGY}

We consider a 2-color multibeam satellite communication system (see Fig. 2) in which beams are paired for the duration of a time slot in order to simultaneously serve two users, one in each of the corresponding spot beam areas. To this end, each beam sends the weighted sum of two symbol sequences. Provided that the beams are judiciously grouped in pairs, the interference from outside the pair can be reduced to a high extent.

Let 1 and 2 denote a pair of beams that simultaneously serves users 1 and 2. Beam 1 sends $\sqrt{\lambda_{1}} s^{(1)}+\sqrt{\bar{\lambda}_{1}} s^{(2)}$ and beam 2 sends $\sqrt{\lambda_{2}} \boldsymbol{s}^{(3)}+\sqrt{\bar{\lambda}_{2}} \boldsymbol{s}^{(4)}$. Here, $\bar{\lambda}_{i}$ is a short-hand notation for $\left(1-\lambda_{i}\right), \lambda_{1}$ and $\lambda_{2}$ are design parameters that take values in $[0,1)$, and $\boldsymbol{s}^{(j)} \in \mathbb{C}^{N \times 1}, j=1,2,3,4$, denote coded symbol sequences of size $N$ with components $s^{(j)}[k]$ that are normalized to unit enery, i.e., $\mathbb{E}\left[\left|s^{(j)}[k]\right|^{2}\right]=1$. Both beams transmit at the same symbol rate $1 / T_{s}$, with the same amount of energy $E_{s}$ per symbol interval and by linearly modulating an identical unit-energy realvalued even square-root Nyquist pulse with respect to the symbol period $T_{s}$. The signal received at UT $\beta, \beta \in\{1,2\}$, can be written as

$$
\begin{aligned}
y_{\beta}(t) & =h_{\beta, 1} \sqrt{\lambda_{1} E_{s}} \sum_{k=1}^{N} s^{(1)}[k] p\left(t-k T_{s}\right) \\
& +h_{\beta, 1} \sqrt{\bar{\lambda}_{1} E_{s}} \sum_{k=1}^{N} s^{(2)}[k] p\left(t-k T_{s}\right) \\
& +h_{\beta, 2} \sqrt{\lambda_{2} E_{s}} \sum_{k=1}^{N} s^{(3)}[k] p\left(t-k T_{s}-\tau_{\beta}\right) \\
& +h_{\beta, 2} \sqrt{\bar{\lambda}_{2} E_{s}} \sum_{k=1}^{N} s^{(4)}[k] p\left(t-k T_{s}-\tau_{\beta}\right) \\
& +w_{\beta}(t) .
\end{aligned}
$$

Here, $h_{\beta, i}$ represents the complex channel coefficient between beam $i$ and UT $\beta, w_{\beta}(t)$ is additive white Gaussian noise with power spectral density $N_{0, \beta}$ and $\tau_{\beta}$ is the TO between beams 1 and 2 at UT $\beta$. Applying the signal received at UT $\beta$ to a matched filter with impulse response $p(t)$ and sampling the filter output at time instants $t=k T_{s}$ and at $t=k T_{s}+\tau_{\beta}$ for $k=1,2, \ldots, N$ (1 sample per symbol and per beam) yields a sufficient statistic for the transmitted symbol vectors $\left(\boldsymbol{s}^{(1)}, \boldsymbol{s}^{(2)}, \boldsymbol{s}^{(3)}, \boldsymbol{s}^{(4)}\right)$ [12]. We have:

$$
\begin{aligned}
& \boldsymbol{z}_{\beta} \\
& =h_{\beta, 1} \sqrt{\lambda_{1}}\left[\begin{array}{c}
\boldsymbol{G}(0) \\
\boldsymbol{G}\left(\tau_{\beta}\right)
\end{array}\right] \boldsymbol{s}^{(1)}+h_{\beta, 1} \sqrt{\bar{\lambda}_{1}}\left[\begin{array}{c}
\boldsymbol{G}(0) \\
\boldsymbol{G}\left(\tau_{\beta}\right)
\end{array}\right] \boldsymbol{s}^{(2)} \\
& +h_{\beta, 2} \sqrt{\lambda_{2}}\left[\begin{array}{c}
\boldsymbol{G}\left(-\tau_{\beta}\right) \\
\boldsymbol{G}(0)
\end{array}\right] \boldsymbol{s}^{(3)}+h_{\beta, 2} \sqrt{\bar{\lambda}_{2}}\left[\begin{array}{c}
\boldsymbol{G}\left(-\tau_{\beta}\right) \\
\boldsymbol{G}(0)
\end{array}\right] \boldsymbol{s}^{(4)} \\
& +\boldsymbol{w}_{\beta},
\end{aligned}
$$

with $\boldsymbol{z}_{\beta} \in \mathbb{C}^{2 N \times 1}, \boldsymbol{w}_{\beta} \in \mathbb{C}^{2 N \times 1}$ a Gaussian noise vector with zero mean and co-variance matrix $\mathbb{E}\left[\boldsymbol{w}_{\beta} \boldsymbol{w}_{\beta}^{H}\right]=$ $\frac{N_{0, \beta}}{E_{s}}\left[\begin{array}{cc}\boldsymbol{G}(0) & \boldsymbol{G}\left(-\tau_{\beta}\right) \\ \boldsymbol{G}\left(\tau_{\beta}\right) & \boldsymbol{G}(0)\end{array}\right], \quad \boldsymbol{G}(\tau) \quad \in \quad \mathbb{C}^{N \times N}$ a Wiener class Toeplitz matrix [13] with components $(\boldsymbol{G}(\tau))_{k, l}=$ $g\left((k-l) T_{s}+\tau\right)$, where $g(t)=\int p(u) p(u-t) d u$ denotes the impulse response of the cascade of the transmit filter $p(t)$ and the receive filter $p^{*}(t)$. In the special case where $\tau_{\beta}$ equals 0 , the first and last $N$ components of $\boldsymbol{z}_{\beta}$ are the same and as a consequence the first $N$ components of $\boldsymbol{z}_{\beta}$ are a sufficient statistic on their own.

The transmitter ( $\mathrm{Tx})$ and receiver $(\mathrm{Rx})$ strategies adopted in the practical SC-SCD and NCRS schemes from [1] can be summarized as follows (see Fig. 3). For SC-SCD:

-Tx: The information for one user is encoded in $\left(s^{(1)}, s^{(3)}\right)$, the information for the other user is encoded in $\left(s^{(2)}, s^{(4)}\right)$.

-Rx: One of the UTs adopts SCD, it decodes and cancels the interference signal components prior to decoding the useful signal components. The other UT only decodes the useful signal components. Without loss of generality we will assume that $\left(\boldsymbol{s}^{(1)}, \boldsymbol{s}^{(3)}\right)$ is intended for the UT that performs SCD.

On the other hand, for NCRS:

-Tx: Private information for UT 1 (2) is encoded in $\boldsymbol{s}^{(1)}\left(\boldsymbol{s}^{(3)}\right)$. Public information is encoded in $\left(s^{(2)}, s^{(4)}\right)$ and can be allocated to either user. ${ }^{3}$,

-Rx: Both UTs adopt SCD; $\left(\boldsymbol{s}^{(2)}, \boldsymbol{s}^{(4)}\right)$ is decoded and canceled prior to decoding $\boldsymbol{s}^{(1)}$ (at UT 1) or $\boldsymbol{s}^{(3)}$ (at UT 2).

\footnotetext{
${ }^{3}$ More generally, the public information can also be split up, in which case $\boldsymbol{s}^{(2)}$ and $\boldsymbol{s}^{(4)}$ are allocated to different users. However, for the simplicity of the exposition such a scenario is not further considered in this paper.
} 


\section{ACHIEVABLE RATE REGION ANALYSIS}

In this section we compute the region of achievable rate pairs $\left(R_{1}, R_{2}\right)$, with $R_{\beta}$ the information rate for user $\beta$, by finding the relevant mutual information rates from (1), when the transmitted symbol sequences are modeled as long sequences of independent unit-energy complex-valued Gaussian random variables as in [1], [7], [12], [14].

In general, the mutual information between two random vector variables $\boldsymbol{x}$ (send) and $\boldsymbol{y}$ (received), expressed in bit per vector $\boldsymbol{x}$, is:

$$
I(\boldsymbol{x} ; \boldsymbol{y})=\mathbb{E}\left[\log _{2}\left(\frac{p(\boldsymbol{y} \mid \boldsymbol{x})}{p(\boldsymbol{y})}\right)\right],
$$

where $\mathbb{E}[$.$] is the expectation over (\boldsymbol{x}, \boldsymbol{y})$. Similarly, the conditional mutual information between $\boldsymbol{x}$ and $\boldsymbol{y}$ given $\boldsymbol{x}^{\prime}$, expressed in bit per vector $\boldsymbol{x}$, is:

$$
I\left(\boldsymbol{x} ; \boldsymbol{y} \mid \boldsymbol{x}^{\prime}\right)=\mathbb{E}\left[\log _{2}\left(\frac{p\left(\boldsymbol{y} \mid \boldsymbol{x}, \boldsymbol{x}^{\prime}\right)}{p\left(\boldsymbol{y} \mid \boldsymbol{x}^{\prime}\right)}\right)\right],
$$

where $\mathbb{E}[$.$] is the expectation over \left(\boldsymbol{x}, \boldsymbol{x}^{\prime}, \boldsymbol{y}\right)$. The mutual information in bit per component of $\boldsymbol{x}$ is then $\mathbb{I}_{\boldsymbol{x} ; \boldsymbol{y}}^{N \rightarrow \infty}=$ $\lim _{N \rightarrow \infty} \frac{1}{N} I(\boldsymbol{x} ; \boldsymbol{y})$ and $\mathbb{I}_{\boldsymbol{x} ; \boldsymbol{y} \mid \boldsymbol{x}^{\prime}}^{N \rightarrow \infty}=\lim _{N \rightarrow \infty} \frac{1}{N} I\left(\boldsymbol{x} ; \boldsymbol{y} \mid \boldsymbol{x}^{\prime}\right)$, respectively, where $N$ is the number of components in $\boldsymbol{x}, \boldsymbol{x}^{\prime}$ and $\boldsymbol{y}$, and $N$ goes to infinity.

In both SC-SCD and NCRS, two symbol sequences are decoded by both UTs while the remaining two symbol sequences are decoded only by the intended UT. In SC-SCD, the latter 2 symbol sequences are intended for the same user, while in NCRS this is not the case. As $\left(\boldsymbol{s}^{(2)}, \boldsymbol{s}^{(4)}\right)$ might be intended for either user, there are two achievable rate regions for given $\left(h_{1,1}, h_{1,2}, h_{2,1}, h_{2,2}, \lambda_{1}, \lambda_{2}, \tau_{1}, \tau_{2}\right)$; the region that corresponds to the case where $\left(\boldsymbol{s}^{(2)}, \boldsymbol{s}^{(4)}\right)$ is intended for user $i$ will be denoted as $\mathcal{R}_{i}(\boldsymbol{H}, \boldsymbol{\lambda}, \boldsymbol{\tau})$, where $i \in\{1,2\}, \boldsymbol{H}=\left[\boldsymbol{h}_{1}^{T} \boldsymbol{h}_{2}^{T}\right]$, with $\boldsymbol{h}_{\beta}=\left[h_{\beta, 1} h_{\beta, 2}\right]^{T}$ for $\beta \in\{1,2\}, \boldsymbol{\lambda}=\left(\lambda_{1}, \lambda_{2}\right)$ and $\boldsymbol{\tau}=\left(\tau_{1}, \tau_{2}\right)$. Using the short-hand notations $\boldsymbol{s}=\left(\boldsymbol{s}^{(1)}, \boldsymbol{s}^{(3)}\right)$ and $\boldsymbol{s}^{\prime}=\left(\boldsymbol{s}^{(2)}, \boldsymbol{s}^{(4)}\right)$, we obtain for SC-SCD:

$$
\left.\begin{array}{l}
\mathcal{R}_{1}(\boldsymbol{H}, \boldsymbol{\lambda}, \boldsymbol{\tau}) \\
=\left\{\begin{array}{c}
\left(R_{1}, R_{2}\right): \\
0 \leq R_{1} \leq \min \left(\mathbb{I}_{\boldsymbol{s}^{\prime} ; \boldsymbol{y}_{1}}^{N \rightarrow \infty}\left(\boldsymbol{h}_{1}, \boldsymbol{\lambda}, \tau_{1}\right), \mathbb{I}_{\boldsymbol{s}^{\prime} ; \boldsymbol{y}_{2}}^{N \rightarrow \infty}\right. \\
0 \leq R_{2} \leq \mathbb{I}_{\boldsymbol{s} ; \boldsymbol{y}_{2} \mid \boldsymbol{s}^{\prime}}^{\left.N \rightarrow \boldsymbol{h}_{2}, \boldsymbol{\lambda}, \tau_{2}\right)}
\end{array}\left(\boldsymbol{h}_{2}, \boldsymbol{\lambda}, \tau_{2}\right)\right),
\end{array}\right\},
$$

$\mathcal{R}_{2}(\boldsymbol{H}, \boldsymbol{\lambda}, \boldsymbol{\tau})$

$$
=\left\{\begin{array}{c}
\left(R_{1}, R_{2}\right): \\
0 \leq R_{1} \leq \mathbb{I}_{\boldsymbol{s} ; \boldsymbol{y}_{1} \mid \boldsymbol{s}^{\prime}}^{\left.N \rightarrow \boldsymbol{h}_{1}, \boldsymbol{\lambda}, \tau_{1}\right)} \\
0 \leq R_{2} \leq \min \left(\mathbb{I}_{\boldsymbol{s}^{\prime} ; \boldsymbol{y}_{1}}^{N \rightarrow \infty}\left(\boldsymbol{h}_{1}, \boldsymbol{\lambda}, \tau_{1}\right), \mathbb{I}_{\boldsymbol{s}^{\prime} ; \boldsymbol{y}_{2}}^{N \rightarrow \infty}\left(\boldsymbol{h}_{2}, \boldsymbol{\lambda}, \tau_{2}\right)\right)
\end{array}\right\},
$$

while for NCRS we have:

$$
\begin{aligned}
& \mathcal{R}_{1}(\boldsymbol{H}, \boldsymbol{\lambda}, \boldsymbol{\tau}) \\
& =\left\{\begin{array}{c}
\left(R_{1}, R_{2}\right): \\
0 \leq R_{1} \leq \min \left(\mathbb{I}_{\boldsymbol{s}^{\prime} ; \boldsymbol{y}_{1}}^{N \rightarrow \infty}\left(\boldsymbol{h}_{1}, \boldsymbol{\lambda}, \tau_{1}\right), \mathbb{I}_{\boldsymbol{s}^{\prime} ; \boldsymbol{y}_{2}}^{N \rightarrow \infty}\right. \\
+\mathbb{I}_{\boldsymbol{s}}^{N \rightarrow \infty} ; \boldsymbol{y}_{1} \mid \boldsymbol{s}^{\prime}\left(\boldsymbol{h}_{1}, \boldsymbol{\lambda}, \tau_{1}\right), \\
\left.0 \leq R_{2} \leq \mathbb{I}_{\boldsymbol{s}^{(3)} ; \boldsymbol{y}_{2} \mid \boldsymbol{s}^{\prime}}^{\left.N \rightarrow \boldsymbol{h}_{2}, \boldsymbol{\lambda}, \tau_{2}\right)}\right)
\end{array}\right\},
\end{aligned}
$$

$$
\begin{aligned}
& \mathcal{R}_{2}(\boldsymbol{H}, \boldsymbol{\lambda}, \boldsymbol{\tau}) \\
& =\left\{\begin{array}{c}
\left(R_{1}, R_{2}\right): \\
0 \leq R_{1} \leq \mathbb{I}_{\boldsymbol{s}^{(1)} ; \boldsymbol{y}_{1} \mid \boldsymbol{s}^{\prime}}^{\left.N \rightarrow \boldsymbol{h}_{1}, \boldsymbol{\lambda}, \tau_{1}\right),} \\
0 \leq R_{2} \leq \min \left(\mathbb{I}_{\boldsymbol{s}^{\prime} ; \boldsymbol{y}_{1}}^{\left.N \rightarrow \boldsymbol{h}_{1}, \boldsymbol{\lambda}, \tau_{1}\right), \mathbb{I}_{\boldsymbol{s}^{\prime} ; \boldsymbol{y}_{2}}^{N \rightarrow \infty}}\left(\boldsymbol{h}_{2}, \boldsymbol{\lambda}, \tau_{2}\right)\right) \\
+\mathbb{I}_{\boldsymbol{s}^{(3)} ; \boldsymbol{y}_{2} \mid \boldsymbol{s}^{\prime}}^{\left.N \rightarrow \boldsymbol{h}_{2}, \boldsymbol{\lambda}, \tau_{2}\right)}
\end{array}\right\} .
\end{aligned}
$$

Relying on the Gaussian character of the symbol sequences and the noise, using the Schur complement and exploiting the asymptotic properties of sequences of Wiener class Toeplitz matrices [12], [13], we obtain the following closed-form expressions for $\mathbb{I}_{\boldsymbol{s}^{\prime} ; \boldsymbol{y}_{\beta}}^{N \rightarrow \infty}\left(\boldsymbol{h}_{\beta}, \boldsymbol{\lambda}, \tau_{\beta}\right), \mathbb{I}_{\boldsymbol{s} ; \boldsymbol{y}_{\beta} \mid \boldsymbol{s}^{\prime}}^{N \rightarrow \infty}\left(\boldsymbol{h}_{\beta}, \boldsymbol{\lambda}, \tau_{\beta}\right), \mathbb{I}_{\boldsymbol{s}^{(1)} ; \boldsymbol{y}_{\beta} \mid \boldsymbol{s}^{\prime}}^{N \rightarrow \infty}$ and $\mathbb{I}_{\boldsymbol{s}^{(3)} ; \boldsymbol{y}_{\beta} \mid \boldsymbol{s}^{\prime}}^{N \rightarrow \infty}, \beta \in\{1,2\}:$

$$
\begin{aligned}
& \mathbb{I}_{\boldsymbol{s}^{\prime} ; \boldsymbol{y}_{\beta}}^{N \rightarrow \infty}\left(\boldsymbol{h}_{\beta}, \boldsymbol{\lambda}, \tau_{\beta}\right) \\
& =T_{s} \int_{-\frac{1}{2 T_{s}}}^{\frac{1}{2 T_{s}}} \log _{2}\left(\frac{\mathcal{G}\left(\gamma_{\beta, 1}, \gamma_{\beta, 2}, f, \tau_{\beta}\right)}{\mathcal{G}\left(\gamma_{\beta, 1} \lambda_{1}, \gamma_{\beta, 2} \lambda_{2}, f, \tau_{\beta}\right)}\right) \mathrm{d} f, \\
& \mathbb{I}_{\boldsymbol{s} ; \boldsymbol{y}_{\beta} \mid \boldsymbol{s}^{\prime}}^{N \rightarrow \infty}\left(\boldsymbol{h}_{\beta}, \boldsymbol{\lambda}, \tau_{\beta}\right) \\
& =T_{s} \int_{-\frac{1}{2 T_{S}}}^{\frac{1}{2 T_{s}}} \log _{2}\left(\mathcal{G}\left(\gamma_{\beta, 1} \lambda_{1}, \gamma_{\beta, 2} \lambda_{2}, f, \tau_{\beta}\right)\right) \mathrm{d} f, \\
& \mathbb{I}_{\boldsymbol{s}^{(1)} ; \boldsymbol{y}_{\beta} \mid \boldsymbol{s}^{\prime}}^{N \rightarrow \infty}\left(\boldsymbol{h}_{\beta}, \boldsymbol{\lambda}, \tau_{\beta}\right) \\
& =T_{s} \int_{-\frac{1}{2 T_{s}}}^{\frac{1}{2 T_{s}}} \log _{2}\left(\frac{\mathcal{G}\left(\gamma_{\beta, 1} \lambda_{1}, \gamma_{\beta, 2} \lambda_{2}, f, \tau_{\beta}\right)}{1+\gamma_{\beta, 2} \lambda_{2}}\right) \mathrm{d} f, \\
& \mathbb{I}_{\boldsymbol{s}^{(3)} ; \boldsymbol{y}_{\beta} \mid \boldsymbol{s}^{\prime}}^{N \rightarrow \infty}\left(\boldsymbol{h}_{\beta}, \boldsymbol{\lambda}, \tau_{\beta}\right) \\
& =T_{s} \int_{-\frac{1}{2 T_{s}}}^{\frac{1}{2 T_{s}}} \log _{2}\left(\frac{\mathcal{G}\left(\gamma_{\beta, 1} \lambda_{1}, \gamma_{\beta, 2} \lambda_{2}, f, \tau_{\beta}\right)}{1+\gamma_{\beta, 1} \lambda_{1}}\right) \mathrm{d} f \text {, }
\end{aligned}
$$

where

$$
\gamma_{i, j}=\frac{E_{s}\left|h_{i, j}\right|^{2}}{N_{0, i}}
$$

and

$$
\begin{aligned}
\mathcal{G}\left(\gamma_{\beta, 1}, \gamma_{\beta, 2}, f, \tau_{\beta}\right) & =1+\gamma_{\beta, 1}+\gamma_{\beta, 2} \\
& +\gamma_{\beta, 1} \gamma_{\beta, 2}\left(1-\left|G\left(e^{j 2 \pi f T_{s}} ; \tau_{\beta}\right)\right|^{2}\right),
\end{aligned}
$$

with

$$
G\left(e^{j 2 \pi f T_{s}} ; \tau\right)=\frac{e^{-j 2 \pi f \tau}}{T_{s}} \sum_{m=-\infty}^{+\infty} G\left(f-\frac{m}{T_{s}}\right) e^{j 2 \pi m \frac{\tau}{T_{s}}},
$$

where $G(f)$ denotes the Fourier transform of $g(t)$. Note that (2)(5) do not depend on the integer part of $\frac{\tau_{\beta}}{T_{s}}$, nor on the sign of $\tau_{\beta}$, nor on the phases of $h_{\beta, 1}$ and $h_{\beta, 2}$ (the latter is one of the appealing features of SC-SCD and NCRS). It follows directly from the Nyquist criterion $\frac{1}{T} \sum_{l} G\left(f-\frac{l}{T}\right)=1$ that we obtain the same result as in [1] when $\tau_{1}$ and $\tau_{2}$ equal 0 . Moreover, it can be shown that $\left|G\left(e^{j 2 \pi f T} ; \tau\right)\right| \leq 1$, with equality for $\tau=0$. As for given $f$ the integrand in (2)-(5) is an increasing function of $\left(1-\left|G\left(e^{j 2 \pi f T} ; \tau\right)\right|^{2}\right)$, the corresponding mutual information is minimum at $\tau=0$. In the special case where $G(f)$ is strictly rectangular with support $f \in\left[-\frac{1}{2 T_{s}}, \frac{1}{2 T_{s}}\right],\left|G\left(e^{j 2 \pi f T_{s}} ; \tau\right)\right|^{2}=1$ independent of the value of $\tau$ such that we also obtain the same result as in [1].

The overall rate region that the system can actually achieve depends on the channel and TO information that is available at the GW transmitter. In [1], it is generally assumed that the GW knows $\gamma_{i, j},(i, j) \in\{1,2\}$. If the $\mathrm{GW}$ also knows $\tau_{i}, i \in\{1,2\}$ 
(which would require additional feedback signaling), the collection of achievable rate pairs is achieved as

$$
\begin{aligned}
& \mathcal{R}(\boldsymbol{H}, \boldsymbol{\tau}) \\
& =\operatorname{conv}\left\{\left(\bigcup_{\boldsymbol{\lambda}} \mathcal{R}_{1}(\boldsymbol{H}, \boldsymbol{\lambda}, \boldsymbol{\tau})\right) \bigcup\left(\bigcup_{\boldsymbol{\lambda}} \mathcal{R}_{2}(\boldsymbol{H}, \boldsymbol{\lambda}, \boldsymbol{\tau})\right)\right\},
\end{aligned}
$$

where conv $(X)$ denotes the convex closure of the set $X$ in the $\left(R_{1}, R_{2}\right)$ plane and $\mathcal{R}(\boldsymbol{H}, \boldsymbol{\tau})>\mathcal{R}(\boldsymbol{H}, \mathbf{0})$ for $\boldsymbol{\tau} \neq \mathbf{0}$. Moreover, in this case the GW can adjust the relative timing of the waveforms such that $\tau$ evolves towards the value that yields the best system performance [14], [15]; this would extend the rate region as follows:

$$
\begin{aligned}
& \mathcal{R}(\boldsymbol{H}) \\
& =\operatorname{conv}\left\{\left(\bigcup_{\boldsymbol{\lambda}, \boldsymbol{\tau}} \mathcal{R}_{1}(\boldsymbol{H}, \boldsymbol{\lambda}, \boldsymbol{\tau})\right) \bigcup\left(\bigcup_{\boldsymbol{\lambda}, \boldsymbol{\tau}} \mathcal{R}_{2}(\boldsymbol{H}, \boldsymbol{\lambda}, \boldsymbol{\tau})\right)\right\} .
\end{aligned}
$$

However, if the transmitter has no information about the TOs $\tau_{1}$ and $\tau_{2}$, the rate selection needs to be performed based on the worst case scenario [12] and therefore the achievable rate region is only $\mathcal{R}(\boldsymbol{H}, \mathbf{0})$, which is the rate region for synchronous symbol reception from [1].

\section{NUMERICAL RESULTS}

In this section, we present numerical results for the rate region $\mathcal{R}(\boldsymbol{H}, \boldsymbol{\tau})$ for SC-SCD and NCRS, assuming a raised cosine pulse $g(t)$ with roll-off $\rho$. In this case, $1-\left|G\left(e^{j 2 \pi f T} ; \tau_{\beta}\right)\right|^{2}$ equals $\sin ^{2}\left(\pi \frac{\tau_{\beta}}{T_{s}}\right) \cos ^{2}\left(\frac{\pi}{\rho}(|f| T-0.5)\right)$, if $\frac{1-\rho}{2 T}<|f|<\frac{1}{2 T}$ and 0 , otherwise.

Fig. 4 and Fig. 5 depict $\mathcal{R}(\boldsymbol{H}, \boldsymbol{\tau}) /(1+\rho)$, which is the achievable rate region in bits per second per Hertz (bps/Hz). Results are presented for several values of $\rho, \gamma=\left(\gamma_{1,1}, \gamma_{1,2}, \gamma_{2,1}, \gamma_{2,2}\right)$ and $\left|\tau_{1}\right|=\left|\tau_{2}\right|=n T_{s}+\epsilon_{\tau} T_{s}$, with $n$ integer and $\epsilon_{\tau} \in[0,0.5]$. Fig. 4 shows $\mathcal{R}(\boldsymbol{H}, \boldsymbol{\tau}) /(1+\rho)$ for SC-SCD and NCRS, $\rho=0.2$, $\epsilon_{\tau}$ equal to 0 or 0.4 and $\gamma=(14 \mathrm{~dB}, 4 \mathrm{~dB}, 4 \mathrm{~dB}, 14 \mathrm{~dB})$. Under these symmetric channel conditions NCRS is known to outperform SC-SCD [1]. For both schemes, the rate region is moderately larger for TOs equal to 0.4 (symbol asynchronous waveform reception) than for TOs equal to 0 (symbol synchronous reception), which is in line with our expectations. Fig. 5 shows $\mathcal{R}(\boldsymbol{H}, \boldsymbol{\tau}) /(1+\rho)$ for SC-SCD, $\gamma=(14 \mathrm{~dB}, 4 \mathrm{~dB}, 14 \mathrm{~dB}, 14 \mathrm{~dB})$ and $\rho \in\{0,0.2,0.5\}$ and $\epsilon_{\tau} \in\{0,0.2,0.5\}$. For given $\rho>0$, the rate region increases if $\epsilon_{\tau}$ increases. The largest rate region is achieved for $\epsilon_{\tau}=0.5$, i.e., the case where the symbols from beam 1 arrive at both receivers precisely half a symbol period later than the symbols from beam 2. An intuitive explanation for this observation is that it is easier to separate two interfering signals as they are received in a more symbol-asynchronous manner; in that case, there is - so to speak the lowest level of mutual interference. Hence, if TO information is available at the $\mathrm{GW}$, it is beneficial to use a synchronization structure that strives for a TO of half a symbol period at both UTs. For given $\epsilon_{\tau}$, the spectral efficiency decreases if $\rho$ increases; however, the gains that are obtained with respect to the case of zero TOs increase for larger values of $\rho$.

\section{CONCLUSIONS AND IDEAS FOR FUTURE WORK}

In this paper, we have computed the achievable rate region for SC-SCD and NCRS [1] in the presence of a non-zero TO between the cooperating beams. Assuming Gaussian signaling, closed-form expressions are obtained for given sets of weight factors, TOs and channel coefficients. It is observed that these expressions do not depend on the integer part of the TOs, nor on the phase of the channel coefficients. It is further shown that for known TOs, the worst case time offset is zero; this is the case where the waveforms

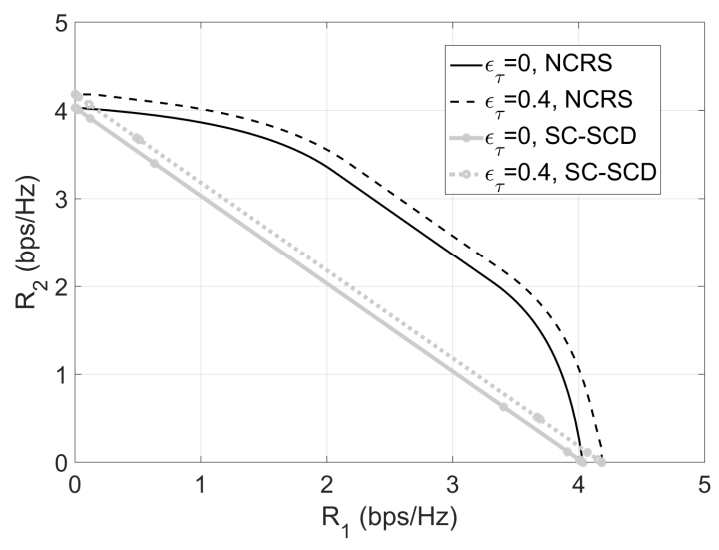

Fig. 4. Rate region in bps/Hz for SC-SCD and NCRS, $\rho=0.2$, $\epsilon_{\tau} \in\{0,0.4\}$ and $\gamma=(14,4,4,14) \mathrm{dB}$.

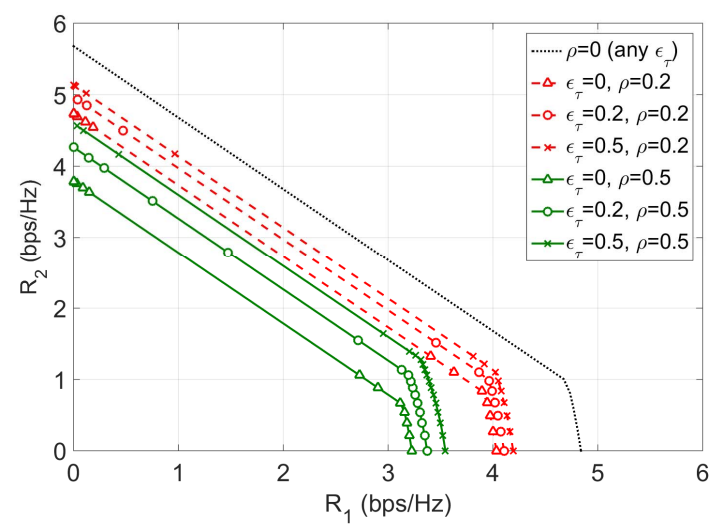

Fig. 5. Rate region in bps/Hz for SC-SCD, $\rho \in\{0,0.2,0.5\}, \epsilon_{\tau} \in$ $\{0,0.2,0.5\}$ and $\gamma=(14,4,14,14) \mathrm{dB}$.

from different beams arrive at the UTs in a symbol-synchronous manner. As a consequence, if no TO information is available at the gateway, the achievable rate region is the same as obtained in [1] where zero TOs are assumed.

To conclude, we point out that, whatever the assumed knowledge at the GW, a system can only achieve the predicted performance if the UTs (i) have complete and accurate channel and timing information at their disposal and (ii) fully exploit this knowledge. Hence, a study on the impact of parameter estimation errors on the achievable rate regions and an analysis of the implementation complexity of potential detectors for SC-SCD and NCRS in the presence of known TOs are interesting topics for follow-up research.

\section{ACKNOWLEDGMENT}

This work has been supported by the European Space Agency funded activity SatNEx IV Contract No.4000113177/15/NL/CLP. The views of the authors of this paper do not reflect the views of ESA. 


\section{REFERENCES}

[1] Màrius Caus, Adriano Pastore, Mònica Novarro, Tomás Ramírez, Carlos Mosquera, Nele Noels, Nader Alagha, and Ana I. Perez-Neira, "Exploratory analysis of superposition coding and rate splitting for multibeam satellite systems," in Proc. 15th Int. Sym. Wireless Commun. Sys. (ISWCS), Lisbon, Portugal, Aug. 2018

[2] Ana I. Perez-Neira, Miguel Angel Vazquez, Sina Maleki, Bhavani Shankar, and Symeon Chatzinotas, "Signal processing for high throughput satellite systems: Challenges in new interference-limited scenarios," http://arxiv.org/abs/1802.03958, Feb. 2018.

[3] Riccardo De Gaudenzi, Nader Alagha, Martina Angelone, and Gennaro Gallinaro, "Exploiting code division multiplexing with decentralized multiuser detection in the satellite multibeam forward link," Int. J. Satell. Commun. Networking, vol. 36, no. 3, pp. 239-276, 2018.

[4] Giuseppe Cocco, Martina Angelone, and Ana I Perez-Neira, "Co-channel interference cancelation at the user terminal in multibeam satellite systems," Int. J. Satell. Commun. Networking, vol. 35, no. 1, pp. 45-65, 2017.

[5] Giulio Colavolpe, Andrea Modenini, Amina Piemontese, and Alessandro Ugolini, "Multiuser detection in multibeam satellite systems: Theoretical analysis and practical schemes," IEEE Trans. Commun., vol. 65, no. 2, pp. 945-955, Feb. 2017.

[6] Alessandro Ugolini, Giulio Colavolpe, and Alessandro Vanelli-Coralli, "A system level approach to the application of multiuser detection in multibeam satellite systems," in Proc. 14th Int. Sym. Wireless Commun. Sys. (ISWCS), Bologna, Italy, Aug. 2017.

[7] Abbas El Gamal and Young-Han Kim, Network information theory, Cambridge University Press, 2011.

[8] Hamdi Joudeh and Bruno Clerckx, "Rate-splitting for maxmin fair multigroup multicast beamforming in overloaded systems," IEEE Trans. Wireless Commun., vol. 16, no. 11, pp. 7276-7289, Nov. 2017.

[9] Pantelis-Daniel Arapoglou, Alberto Ginesi, Stefano Cioni, Stefan Erl, Federico Clazzer, Stefano Andrenacci, and Alessandro Vanelli-Coralli, "DVB-S2X-enabled precoding for high throughput satellite systems," Int. J. Satell. Commun. Networking, vol. 34, no. 3, pp. 439-455, 2016.

[10] ETSI EN 302 307-2 V1.1.1 Digital Video broadcast (DVB), "Implementation guidelines for the second generation systems for broadcasting, interactive services, news gathering, and other broadband satellite aapplication; part 2 - S2 Extensions $(D V B-S 2 X)^{\prime \prime}$, Mar. 2015.

[11] Stefano Andrenacci, Symeon Chatzinotas, Alessandro Vanelli-Coralli, Stefano Cioni, Alberto Ginesi, and Bjorn Ottersten, "Exploiting orthogonality in DVB-S2X through timing pre-compensation," in Proc. 8th Advanced Satell. Multimedia Sys. Conf. and 14th Sig. Process. Space Commun. Workshop (ASMS/SPSC), Palma de Mallorca, Spain, Sept. 2016.

[12] Sergio Verdú, "The capacity region of the symbolasynchronous Gaussian multiple-access channel," IEEE Trans. Inf. Theory, vol. 35, no. 4, pp. 733-751, July 1989.

[13] Robert M. Gray et al., "Toeplitz and circulant matrices: A review," Foundations and Trends in Commun. Inf. Theory, vol. 2, no. 3, pp. 155-239, 2006

[14] Jianxiong Cui, Guangliang Dong, Shengli Zhang, Haitao Li, and Guinian Feng, "Asynchronous NOMA for downlink transmissions," IEEE Commun. Lett., vol. 21, no. 2, pp. 402405, Feb. 2017.

[15] Huseyin Haci, Huiling Zhu, and Jiangzhou Wang, "Performance of non-orthogonal multiple access with a novel asynchronous interference cancellation technique," IEEE Trans Commun, vol. 65, no. 3, pp. 1319-1335, Mar. 2017. 